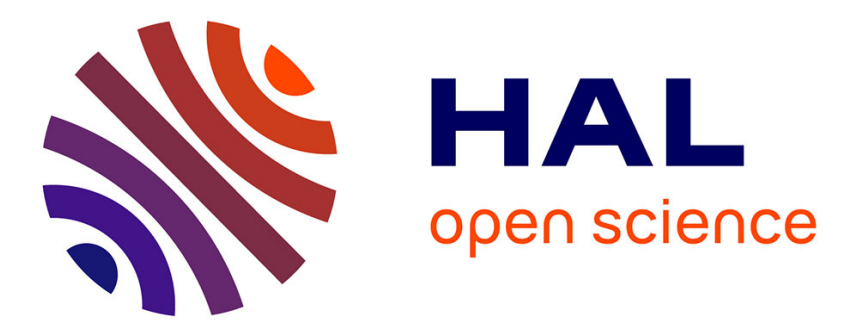

\title{
Methodology for numerical simulations of ellipsoidal particle-laden flows
}

Baptiste Lambert, Lisl Weynans, Michel Bergmann

\section{To cite this version:}

Baptiste Lambert, Lisl Weynans, Michel Bergmann. Methodology for numerical simulations of ellipsoidal particle-laden flows. International Journal for Numerical Methods in Fluids, 2020, 10.1002/fld.4809 . hal-02497079

\section{HAL Id: hal-02497079 \\ https://hal.inria.fr/hal-02497079}

Submitted on 10 Mar 2020

HAL is a multi-disciplinary open access archive for the deposit and dissemination of scientific research documents, whether they are published or not. The documents may come from teaching and research institutions in France or abroad, or from public or private research centers.
L'archive ouverte pluridisciplinaire HAL, est destinée au dépôt et à la diffusion de documents scientifiques de niveau recherche, publiés ou non, émanant des établissements d'enseignement et de recherche français ou étrangers, des laboratoires publics ou privés. 


\section{Methodology for Numerical Simulations of Ellipsoidal Particle-Laden Flows}

\section{B. Lambert $^{1} \quad$ L. Weynans ${ }^{1,2} \quad$ | M.Bergmann ${ }^{1,2}$}

${ }^{1}$ Univ. Bordeaux, IMB, UMR 5251, F-33400 Talence, France

${ }^{2}$ CNRS, IMB, UMR 5251, F-33400 Talence, France

\section{Correspondence}

Email: baptiste.lambert@inria.fr

Funding information
Despite being relevant in many natural and industrial processes, suspensions of non-spherical particles have been largely under-investigated compared to the extensive analyses made on the gravity-driven motions of spherical particles. One of the main reasons for this disparity is the difficulty of accurately correcting the short-range hydrodynamic forces and torques acting on complex particles. These effects, also known as lubrication, are essential to the suspension of the particles and are usually poorly captured by direct numerical simulation of particle-laden flows. In this paper, we propose a partitioned VP-DEM solver which estimates the unresolved hydrodynamic forces and torques. Corrections are made locally on the surface of the interacting particles without any assumption on the particle global geometry. Numerical validations have been made using ellipsoidal particles immersed in an incompressible NavierStokes flow.

\section{KEYWORDS}

Particle Laden Flows, Ellipsoids, Local Lubrication Correction Model, Coupled VP-DEM Solver, 


\section{1 | INTRODUCTION}

Sedimentation is a two-phase flow that appears in various industrial applications as well as in natural flows. For instance, sediments transport in rivers or near-coast influences the flow. By understanding the dynamics of the bed-load transport, the evolution of the river-stream can be forecast to prevent abnormal erosion or obstruction of waterways ${ }^{[1,2]}$. Macroscopic suspensions are also relevant in several industrial applications, such as nuclear waste processing, water treatment ${ }^{[3,4]}$, slurry transportation ${ }^{[5]}$, reinforced plastics manufacturing, or the animation industry ${ }^{[6,7]}$. For dense mixtures such as uncured solid rocket fuel or concretes ${ }^{[8]}$, a high concentration of solid particles is desired without compromising the rheological properties and the flowing behavior of the mixture. Accurate numerical methods are then valuable supports to optimize manufacturing processes.

Most of the numerical methods developed over the past decades focused on suspensions of spherical particles. Extensive experimental and numerical studies of suspensions have highlighted the particle dynamics sensitivity to short range hydrodynamic interactions also known as lubrication effects ${ }^{[9]}$. Often, the particles of interest are non-spherical (ellipsoidal or rod-like, for instance). Furthermore, anisometric solid inclusions interact hydrodynamically much more strongly than spheres do at the same volume fraction ${ }^{[10]}$. Therefore, specific numerical methods need to be developed to accurately simulate lubrication effects, especially in dense suspensions.

The first attempts to simulate non-spherical suspensions used microscale methods without proper lubrication corrections ${ }^{[11]}$, which limited their application to diluted suspension. A simple approach to correct lubrication forces is to model a non-spherical particle as an agglomerate of spherical particles (multi-blob) ${ }^{[7]}$. Hence, non-spherical particle-laden flows can be simulated using robust and efficient solvers initially developed for spherical particles. As the true particle surfaces are not represented, the particle hydrodynamics are roughly approximated. Therefore, lubrication models specifically designed for non-spherical particles are needed to improve the correction accuracy. In the limit where the Reynolds number tends to zero, adaptations of the Stokesian dynamics ${ }^{[12]}$ can be made using theoretical lubrication forces given locally by Cox ${ }^{[13]}$. The resulting methods ${ }^{[10,14]}$ take also into account the many-body interactions which are non-neglectable in dense particle flows. However, the accuracy of this approach comes with a high computational cost and can require the tabulation of several parameters. An alternative to bypass these additional costs is to approximate the surfaces of interacting particles around their contact points by the surfaces of virtual spheres. Lubrication corrections are then estimated using the virtual spheres ${ }^{[15,16]}$.

In this paper, we extend our local lubrication correction model ${ }^{[17]}$ to non-Brownian suspensions of ellipsoidal particles. This lubrication model uses virtual spheres to evaluate local lubrication corrections instead of the global corrections found in the classical lubrication theory ${ }^{[15,16]}$. This article is organized as follows. In Sec. 2, we present the volume penalization method used to solve the incompressible Navier-Stokes equations. Section 3 details the resolution of the particle dynamics using the discrete element method. Finally, our local lubrication correction model is compared to other lubrication models and validated numerically on several benchmarks in the last section. Currently, there is to our knowledge a lack of experimental or numerical data estimating quantitatively the behaviour of lubrication forces in the case of ellipsoidal particles. Consequently we do not dispose of references to compare our numerical results. This fact also enhances the need for accurate models to simulate these phenomenons.

\section{2 | NUMERICAL RESOLUTION OF THE GOVERNING EQUATIONS.}




\section{$D$}

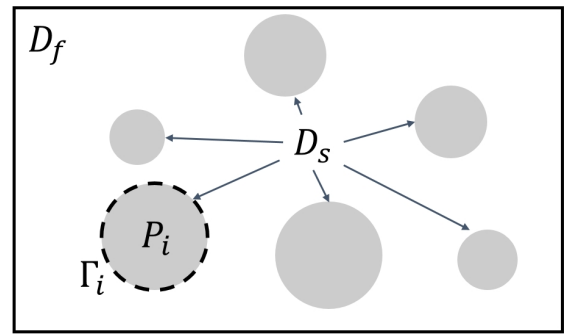

FIGURE 1 Sketch of the computational the domain. The whole $D$ is divided into a fluid domain $D_{\mathrm{f}}$ and solid particles domain $D_{\mathrm{s}}$.

The governing equations considered are the incompressible Navier-Stokes equations for a viscous fluid:

$$
\left\{\begin{aligned}
\frac{\partial u_{i}}{\partial t}+\frac{\partial\left(u_{i} u_{j}\right)}{\partial x_{j}} & =-\frac{1}{\rho} \frac{\partial p}{\partial x_{i}}+\frac{\mu}{\rho} \frac{\partial}{\partial x_{j}}\left(\frac{\partial u_{i}}{\partial x_{j}}\right)+\chi \lambda\left(u_{\tau, i}-u_{i}\right), \\
\frac{\partial u_{i}}{\partial x_{i}} & =0
\end{aligned}\right.
$$

where $(i ; j)=\{1,2,3\}^{2}, u_{i}$ are the velocity components and $p$ is the pressure field. The density and dynamic viscosity of the Newtonian fluid are denoted by $\rho$ and $\mu$, respectively. The system (1) is solved in the three dimensional domain $D=D_{\mathrm{f}} \cup D_{\mathrm{s}}$ using a uniform Cartesian mesh, where $N$ spherical particles $P_{i}$ form the solid domain $D_{\mathrm{s}}=\bigcup_{i=1}^{N} P_{i}$ with $P_{i} \cap P_{j}=\varnothing$ for $i \neq j$ and $D_{\mathrm{f}}=D \backslash D_{\mathrm{s}}$ is the fluid domain. The interface between the solid and fluid phases is denoted $\Gamma_{\mathrm{s}}=\bigcup_{i=1}^{N} \Gamma_{i}$. Each particle $P_{i}$ is assumed to be homogeneous with a density $\rho_{\mathrm{p}, i}$.

A no-slip boundary condition is implicitly imposed at the interface $\Gamma_{s}$, by the penalty term $\chi \lambda\left(u_{\tau, i}-u_{i}\right)$, where $\lambda$ is the porosity and $\chi$ is the particle characteristic function (see Sec. 3.1). Indeed, as reminded by Angot et al. ${ }^{[18]}$, solving the penalized Eqs. (1) is equivalent to solve the incompressible Navier-Stokes equations in the fluid domain and to enforce a no-slip boundary condition at the boundary $\Gamma_{s}$ when $\lambda \rightarrow+\infty$. After the penalization step, solid velocity is enforced on all grid elements fully included inside particles while the velocity on fluid grid elements is not modified.

\section{1 | Discretization of the governing equations.}

The Navier-Stokes equations (1) are discretized on a uniform Cartesian mesh using a cell-centered collocated arrangement of the primitive variables $\left(p, u_{i}\right)$. Face-centered velocities $v_{i}$ are also introduced in addition to the cellcentered velocities $u_{i}$, to eliminate odd-even decoupling which can lead to large pressure variations in space ${ }^{[19]}$. The equations are integrated in time using a classical projection scheme introduced by Chorin ${ }^{[20]}$ and Temam ${ }^{[21]}$. The detailed numerical resolution of the system of Eqs. 1 can be found in our previous works ${ }^{[22,17]}$. 


\section{3 | DYNAMICS OF THE PARTICLES.}

The particle dynamics is solved by a discrete element method (DEM) which is primarily devoted to multi-contact interactions for a large suspension ${ }^{[23]}$. The dynamics of each rigid particle is obtained by the Newton-Euler equations:

$$
\begin{gathered}
m_{i} \frac{d \boldsymbol{U}_{i}}{d t}=\boldsymbol{F}_{i}^{\mathrm{hyd}}+\boldsymbol{F}_{i}^{\mathrm{coll}}+\boldsymbol{F}_{i}^{\mathrm{ext}}, \\
\frac{d J_{i} \boldsymbol{\Omega}_{i}}{d t}=\boldsymbol{T}_{i}^{\mathrm{hyd}}+\boldsymbol{T}_{i}^{\mathrm{coll}},
\end{gathered}
$$

for a given particle $P_{i}$ of mass $m_{i}$, inertia matrix $J_{i}$, linear velocity $U_{i}$ of the mass center and rotational velocity $\boldsymbol{\Omega}_{i}$. The hydrodynamic forces and torques are respectively denoted $\boldsymbol{F}_{i}^{\text {hyd }}$ and $\boldsymbol{T}_{i}^{\text {hyd }}$ (see Sec. 3.3). Non-hydrodynamic forces like gravity (which is the only external force considered here) are denoted $\boldsymbol{F}_{i}^{\text {ext }}$. The force $\boldsymbol{F}_{i}^{\text {coll }}$ and torque $\boldsymbol{T}_{i}^{\text {coll }}$ represent the effects of solid contacts of $P_{i}$ with obstacles (see Sec. 3.5). The position of the mass center and the orientation angle of the particle are given by $\boldsymbol{X}_{i}$ and $\boldsymbol{\Theta}_{i}$ and are simply computed from the velocities $\boldsymbol{U}_{i}$ and $\boldsymbol{\Omega}_{i}$.

An ellipsoidal particle surface is defined by all $(x, y, z) \in \mathbb{R}^{3}$ such that:

$$
\frac{x^{2}}{a^{2}}+\frac{y^{2}}{b^{2}}+\frac{z^{2}}{c^{2}}=1
$$

where $a, b$, and $c$ are the three semi-axes of the ellipsoid (Fig. 2). The particle is called a spheroid when $b=c$. For spheroidal particles, the particle aspect ratio is defined as $\mathcal{A} \mathcal{R}=a / b$. The spheroid is an oblate if $\mathcal{A} \mathcal{R}<1$, a prolate if $\mathcal{A} \mathcal{R}>1$ and a sphere otherwise. The equivalent sphere, of diameter $D_{\mathrm{eq}}=2 \sqrt[3]{a b c}$, is the sphere with the same volume as the particle.

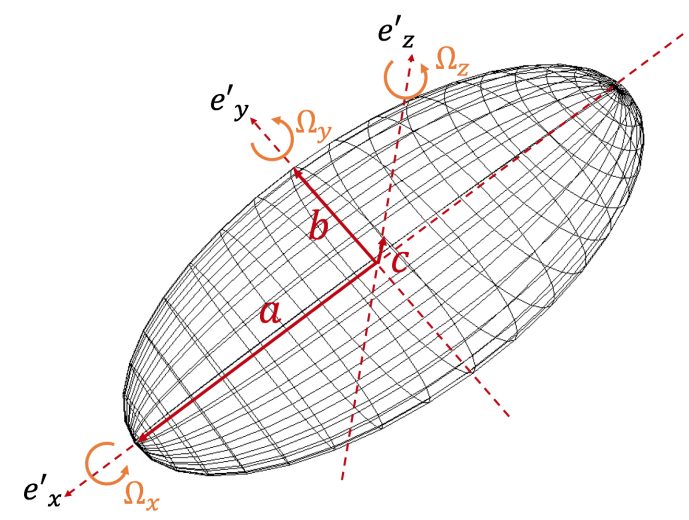

FIGURE 2 Representation of the particle with surface mesh in coordinate system $\left(\boldsymbol{e}_{\boldsymbol{x}}^{\prime}, \boldsymbol{e}_{\boldsymbol{y}}^{\prime}, \boldsymbol{e}_{\boldsymbol{z}}^{\prime}\right)$.

The surface of each particle is meshed with $N_{p}$ elements (Fig. 2). These meshes are used to compute the level-set function and the hydrodynamic forces acting on the particle (Sec. 3.3).

Before going into detail on how each force and torque acting on the particles are computed, two non trivial problems 
need to be addressed, since the particles are not necessary spherical. First, the global level-set function needs to be computed to locate the particles on the background grid and perform the penalization. Subsequently, the distance between a particle to an obstacle (wall or particle) has to be accurately and efficiently computed for the lubrication and collision models.

\section{1 | Computation of the global level-set.}

The global level-set function $\mathcal{L} \mathcal{S}$ is defined on all cells of the background grid (where the penalized Navier-Stokes equations are solved) as the minimal signed distance of the given cell center to the particle surfaces. The level-set is positive on fluid cells and negative on solid cells.

For spherical particles, the level-set at the cell center $\boldsymbol{X}$ is given by

$$
\mathcal{L} \mathcal{S}(\boldsymbol{X})=\min _{i \in \llbracket 1, N \rrbracket}\left(\left\|\boldsymbol{X}-\boldsymbol{X}_{i}\right\|-a_{i}\right)
$$

Unfortunately, the ellipsoid surfaces are not easily characterized in the global coordinate system. Therefore, the level-set is computed via the particle surface mesh. The level-set at a given cell center $\boldsymbol{x}$ is computed as follows:

1. The closest particle surface element is found among all the particles of the system.

2. The position $\boldsymbol{x}$ is projected on the closest particle surface element. This orthogonal projection is denoted $\boldsymbol{X}_{\Gamma}$.

3. The level-set $\mathcal{L} \mathcal{S}(\boldsymbol{x})$ is then given by $\left\|\boldsymbol{x}-\boldsymbol{X}_{\Gamma}\right\|$ and the sign of $\mathcal{L} \mathcal{S}(\boldsymbol{x})$ is positive if $\boldsymbol{x}$ is inside the particle. Otherwise, the sign of $\mathcal{L} \mathcal{S}(\boldsymbol{x})$ is negative.

The particles are located on the grid by the characteristic function $\chi=1_{D_{s}}$. Hence, $\chi$ is defined on each cell center location $\boldsymbol{x}$ such that $\chi(\boldsymbol{x})=1$ if $\mathcal{L} \mathcal{S}(\boldsymbol{x}) \leq 0$, and $\chi(\boldsymbol{x})=0$ otherwise. Simulations could be made more cost-efficient by implementing a method which would balance computation and transport of the level-set.

\subsection{Computation of the minimal distance between two bodies.}

When all the particles are spherical, the computation of the minimal distance between a particle and a wall or another particle is straightforward ${ }^{[17,24]}$. However, the problem becomes more complex if ellipsoidal particles are considered. The localization of the contact points for a pair of particles can be efficiently computed by the following iterative procedure $^{[25]}$ (see Fig. 3):

1. The search algorithm starts from two arbitrary points $\left(\boldsymbol{X}_{\mathrm{c}, i}, \boldsymbol{X}_{\mathrm{c}, j}\right)^{k}$ on the surface of the two particles. These two points are assumed to be the nearest points to the other particle surface.

2. Two spheres are constructed completely inside the ellipsoids such that the sphere and ellipsoid surfaces are tangent at the current nearest points $\left(\boldsymbol{X}_{\mathrm{c}, i}, \boldsymbol{X}_{\mathrm{c}, j}\right)^{k}$.

3. A new guess of the contact points $\left(\boldsymbol{X}_{\mathrm{c}, i}, \boldsymbol{X}_{\mathrm{c}, j}\right)^{k+1}$ is then found by the intersection of the line generated by the centers of the two spheres and the ellipsoid surfaces.

4. If not converged, go back to step 2. Convergence is obtained when the line generated by the centers of the two spheres is co-linear with the ellipsoid surface gradients at the contact points $\left(\boldsymbol{X}_{\mathrm{c}, i}, \boldsymbol{X}_{\mathrm{c}, j}\right)^{k+1}$.

The procedure converges faster as the radius of the virtual spheres increases, and the initial guesses of $\left(\boldsymbol{X}_{\mathrm{c}, i}, \boldsymbol{X}_{\mathrm{c}, j}\right)^{k}$ are 
close to the contact points.

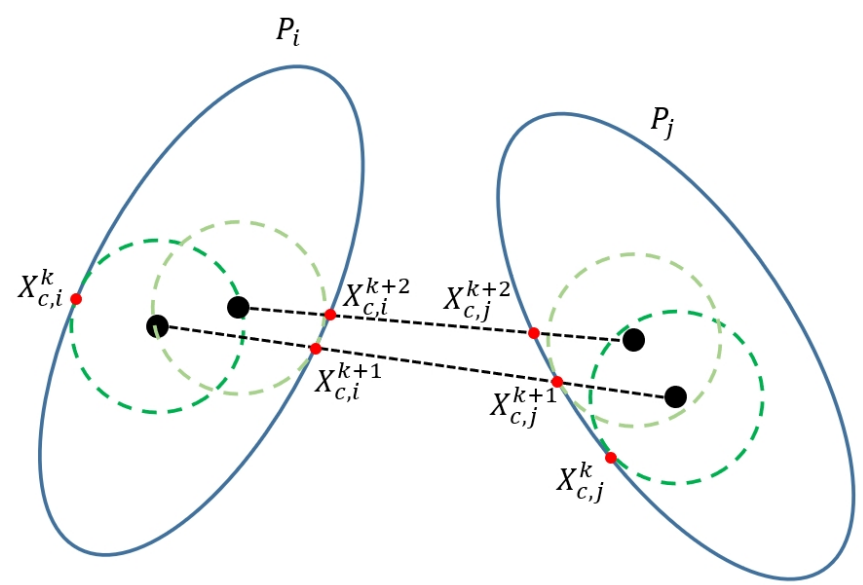

F IGURE 3 Two-dimensional sketch of the iterative method of Lin et al. ${ }^{[25]}$ used to compute the distance between two ellipsoids.

The computation of the minimal distance for a pair particle-wall is more straightforward than for a pair of particles. Indeed, the outgoing normal unit vector of $\Gamma_{i}$ at the contact point $\boldsymbol{X}_{\mathrm{c}, i}$ is co-linear and has an opposite sign to the wall outgoing unit surface vector. Hence, the contact point $\boldsymbol{X}_{\mathrm{c}, i}=\left(x_{\mathrm{c}, i}, y_{\mathrm{c}, i}, z_{\mathrm{c}, i}\right)$ is given by

$$
\left\{\begin{array}{l}
x_{\mathrm{c}, i}=-x_{\mathrm{w}} \frac{a_{i}^{2}}{2}, \\
y_{\mathrm{c}, i}=-y_{\mathrm{w}} \frac{b_{i}^{2}}{2}, \\
z_{\mathrm{c}, i}=-z_{\mathrm{w}} \frac{c_{i}^{2}}{2},
\end{array}\right.
$$

with $e_{\mathrm{w}}=\left(x_{\mathrm{w}}, y_{\mathrm{w}}, z_{\mathrm{W}}\right)$ the wall outgoing unit surface vector in the particle coordinate system.

For non-spherical particles, the collision model (Sec. 3.5) and lubrication correction models (Sec. 3.4.1 and 3.4.2) are evaluated indirectly via virtual spheres. These virtual spheres are defined such that the sphere surfaces fit as much as possible the true particle surfaces at the contact points. For ellipsoids $P_{i}$, the radius of the virtual sphere $R_{\mathrm{vs}, i}$ is given by the Gaussian radius of curvature ${ }^{[15]}$ at the contact point $\boldsymbol{X}_{\mathrm{c}, i}=\left(x_{\mathrm{c}, i}, y_{\mathrm{c}, i}, z_{\mathrm{c}, i}\right)$ :

$$
R_{\mathrm{vs}, i}^{2}=\frac{\left(a_{i}^{4} b_{i}^{4}+b_{i}^{4}\left(c_{i}^{2}-a_{i}^{2}\right) x_{\mathrm{c}, i}^{2}+a_{i}^{4}\left(c_{i}^{2}-b_{i}^{2}\right) y_{\mathrm{c}, i}^{2}\right)^{2}}{a_{i}^{6} b_{i}^{6} c_{i}^{2}}
$$

Subsequently, the center of the virtual sphere $\boldsymbol{X}_{\mathrm{vs}, i}$, in the particle coordinate system, is defined as

$$
\boldsymbol{X}_{\mathrm{vs}, i}=\boldsymbol{X}_{\mathrm{c}, i}-R_{\mathrm{vs}, i} N_{\mathrm{c}, i}
$$


where $\boldsymbol{N}_{\mathrm{c}, i}$ is the outgoing normal unit vector of $\Gamma_{i}$, in the particle coordinate system, at the contact point $\boldsymbol{X}_{\mathrm{c}, i}$. The coordinates of the virtual sphere center are then projected in the global coordinate system using the particle rotation matrix.

\section{3 | Particle hydrodynamics.}

To numerically compute the hydrodynamic force and torque acting on the particle $P_{i}$, the surface $\Gamma_{i}$ is meshed using $N_{\mathrm{p}, i}$ elements. The k-th element of the mesh of $\Gamma_{i}$ is denoted $P_{i}^{k}$ and $s_{k}$ is its surface. The set $L\left(P_{i}^{k}\right)$ is defined as all the particles $P_{j}, j \neq i$ such that the distance between the surface of $P_{j}$ and the center of $P_{i}^{k}$ is lower than $a \epsilon_{\text {lub }}$. The distance $a \epsilon_{\text {lub }}$ is defined as the narrowest gap width between the center of the $P_{i}^{k}$ and a potential nearby obstacle for the solver to fully resolve hydrodynamic interactions.

The total hydrodynamic force and torque on the particle $P_{i}$ are given by

$$
\left\{\begin{array}{c}
\boldsymbol{F}_{i}^{\text {hyd }}=\boldsymbol{F}_{i}^{\text {solv }}+\boldsymbol{F}_{i}^{\mathrm{deg}}, \\
\boldsymbol{T}_{i}^{\text {hyd }}=\boldsymbol{T}_{i}^{\text {solv }}+\boldsymbol{T}_{i}^{\mathrm{deg}} .
\end{array}\right.
$$

The force $\boldsymbol{F}_{i}^{\text {deg }}$ and torque $\boldsymbol{T}_{i}^{\mathrm{deg}}$ are defined as:

$$
\left\{\begin{aligned}
\boldsymbol{F}_{i}^{\mathrm{deg}} & =\int_{\substack{\boldsymbol{p} \in \Gamma_{i}, L(\boldsymbol{p} \neq \varnothing}}(\sigma \cdot \boldsymbol{n}) \mathrm{d} S, \\
\boldsymbol{T}_{i}^{\mathrm{deg}} & =\int_{\substack{\boldsymbol{p} \in \Gamma_{i}, L(\boldsymbol{p}) \neq \varnothing}} a_{i} \boldsymbol{n} \times(\sigma \cdot \boldsymbol{n}) \mathrm{d} S .
\end{aligned}\right.
$$

These two components of the hydrodynamics are underestimated by the numerical simulation due to the insufficient number of grid elements in the gap between the particle $P_{i}$ and its surrounding obstacles to properly capture the fluid flow. Mesh refinement techniques could be considered to improve the resolution of the interstitial flow. However, these methods have a non-negligible computation cost and cannot provide a grid element small enough to accurately solve the interstitial flow down to the potential collision of the particle with the obstacle. Therefore, a lubrication correction model is introduced to balance the degraded hydrodynamics (see Sec. 3.4). Lubrication correction on the force and torque are denoted $\boldsymbol{F}_{i}^{\text {lub }}$ and $\boldsymbol{T}_{i}^{\text {lub }}$.

The remaining of the hydrodynamics, $\boldsymbol{F}_{i}^{\text {solv }}$ and $\boldsymbol{T}_{i}^{\text {solv }}$ are obtained via the flow solver, as follows:

$$
\left\{\begin{aligned}
\boldsymbol{F}_{i}^{\text {solv }} & =\int_{\substack{\boldsymbol{p} \in \Gamma_{i}, L(\boldsymbol{p})=\varnothing}}(\sigma \cdot \boldsymbol{n}) \mathrm{d} S, \\
\boldsymbol{T}_{i}^{\text {solv }} & =\int_{\substack{\boldsymbol{p} \in \Gamma_{i}, L(\boldsymbol{p})=\varnothing}} a_{i} \boldsymbol{n} \times(\sigma \cdot \boldsymbol{n}) \mathrm{d} S .
\end{aligned}\right.
$$

The resolved hydrodynamics are computed by numerical integration of the fluid stress $\sigma$ acting on all elements $P_{i}^{k}$ far 
enough to nearby obstacles:

$$
\begin{cases}\boldsymbol{F}_{i}^{\mathrm{solv}} \approx & \sum_{\substack{k \in \llbracket 1, N_{\mathrm{p}, i} \rrbracket, L\left(P_{i}^{k}\right)=\varnothing}}(\sigma \cdot \boldsymbol{n}) s_{k}, \\ \boldsymbol{T}_{i}^{\text {solv }} \approx & \sum_{\substack{k \in \llbracket 1, N_{\mathrm{p}, i} \rrbracket, L\left(P_{i}^{k}\right)=\varnothing}} a_{i} \boldsymbol{n} \times(\sigma \cdot \boldsymbol{n}) s_{k},\end{cases}
$$

The fluid stress $\sigma$ is interpolated from the pressure and velocities $\left(p, \boldsymbol{u}_{i}\right)$, using a second-order Lagrange scheme at the center of $P_{i}^{k}$.

\section{4 | Lubrication Correction Models}

The accuracy of the computed hydrodynamic forces acting on a particle directly depends on the accuracy of the computation of the fluid flow surrounding the given particle. In dense suspensions, interstitial flows are often poorly resolved by the direct numerical resolution of the Navier-Stokes equations as the gap between interacting particles can be smaller than the grid resolution. To balance the unresolved hydrodynamics, lubrication correction models are introduced. In this section two methods to approximate $\boldsymbol{F}_{i}^{\mathrm{deg}}$ and $\boldsymbol{T}_{i}^{\mathrm{deg}}$ are described.

\subsubsection{Costa Lubrication Correction Model (CLM).}

The $C \mathrm{LM}^{[26]}$ is a two-parameter model which corrects the normal component of the lubrication force on a spherical particle. The correction is made by adding $\Delta \boldsymbol{F}_{i}^{\text {lub }}=\Delta F_{i}^{\text {lub }} \boldsymbol{e}_{\boldsymbol{n}}$ to the computed hydrodynamic force:

$$
\frac{\Delta F_{i}^{\text {lub }}}{6 \pi \mu a U_{i}^{\text {sq }}}= \begin{cases}\lambda\left(\epsilon_{\Delta x}\right)-\lambda(\epsilon), & \epsilon_{\mathrm{col}} \leq \epsilon<\epsilon_{\Delta x}, \\ \lambda\left(\epsilon_{\Delta x}\right)-\lambda\left(\epsilon_{\mathrm{col}}\right), & 0 \leq \epsilon<\epsilon_{\mathrm{col}}, \\ 0, & \text { otherwise }\end{cases}
$$

where $e_{n}$ is defined as represented in Fig. 4. The Stokes amplification factor ${ }^{[27]} \lambda$ is defined for the lubrication interaction between a sphere and a wall as $\lambda_{\mathrm{pw}}$, and for interaction between two spheres as $\lambda_{\mathrm{pp}}$ :

$$
\left\{\begin{array}{l}
\lambda_{\mathrm{pw}}(\epsilon)=\frac{1}{\epsilon}-\frac{1}{5} \ln (\epsilon)-\frac{1}{21} \epsilon \ln (\epsilon)+O(1) \\
\lambda_{\mathrm{pp}}(\epsilon)=\frac{1}{2 \epsilon}-\frac{9}{20} \ln (\epsilon)-\frac{3}{56} \epsilon \ln (\epsilon)+O(1)
\end{array}\right.
$$

Hence, the total hydrodynamic force is given by

$$
\boldsymbol{F}_{i}^{\text {hyd }}=\int_{\Gamma_{i}}(\sigma \cdot \boldsymbol{n}) \mathrm{d} S+\Delta \boldsymbol{F}_{i}^{\text {lub }} .
$$

By this approach, only the dominant component of the lubrication force is corrected, which does not create any torque. Hence, $\boldsymbol{T}_{i}^{\mathrm{lub}}=0$ and $\boldsymbol{T}_{i}^{\mathrm{deg}}$ is assumed neglectable.

The value of the parameter $\epsilon_{\Delta x}$ is determined by simulating the slow approach of a sphere toward a wall or a second particle, for a given grid cell resolution $h$. Simulations without lubrication correction are compared to the analytical solutions given by Brenner ${ }^{[28]}$ and Cooley et al. ${ }^{[29]}$. The parameter $\epsilon_{\Delta x}$ is defined as the largest value of $\epsilon$ such that for 
$\epsilon \leq \epsilon_{\Delta x}$ the hydrodynamic force from the simulation without lubrication correction no longer matches the analytical solution. The CLM parameter is $\epsilon_{\Delta x}=0.06$ for $h=1 / 40$ grid resolution ${ }^{[17]}$. The CLM has been initially designed for spherical before being extended to spheroidal suspensions by Ardekani et. al ${ }^{[15]}$. Their works have shown that the extended CLM balances correction accuracy and computation cost, making one of the most reliable lubrication model for large scale simulations of non spherical particle-laden flows ${ }^{[30]}$.

\subsection{2 | The Local Lubrication Correction Model (LLCM).}

As previously mention, the LLCM has already been introduced in our former publications ${ }^{[17,22]}$ limited to spherical particles. The LLCM relied on a local description of the classical lubrication theory, which is independent of the particle global geometry. Hence, local expressions of the lubrication corrections are identical for spherical and non-spherical particles. However, the latter geometry requires the approximation of the particle surface around the contact points by virtual spherical cap in order to evaluate the lubrication corrections.

The lubrication force $\boldsymbol{F}_{i}^{\text {lub }}$ and torque $\boldsymbol{T}_{i}^{\text {lub }}$ acting on $P_{i}$ are given by

$$
\begin{aligned}
& \boldsymbol{F}_{i}^{\text {lub }}=\sum_{j \in \llbracket 1, N \rrbracket \backslash\{i\}} \boldsymbol{F}_{i, j}^{\text {lub }}+\boldsymbol{F}_{i, \text { wall }}^{\text {lub }}, \\
& \boldsymbol{T}_{i}^{\text {lub }}=\sum_{j \in \llbracket 1, N \rrbracket \backslash\{i\}}^{T_{i, j}^{\text {lub }}+\boldsymbol{T}_{i, \text { wall }}^{\text {lub }},}
\end{aligned}
$$

where $\boldsymbol{F}_{i, j}^{\mathrm{lub}}$ and $\boldsymbol{T}_{i, j}^{\text {lub }}$ are the lubrication force and torque acting on $P_{i}$, created by the interaction of $P_{i}$ and $P_{j}$. The lubrication force and torque created by the interaction of the particle $P_{i}$ with a wall are denoted $\boldsymbol{F}_{i \text {,wall }}^{\text {lub }}$ and $\boldsymbol{T}_{i, \text { wall }}^{\text {lub }}$, and are equivalent to the asymptotic case $\beta \rightarrow+\infty$.

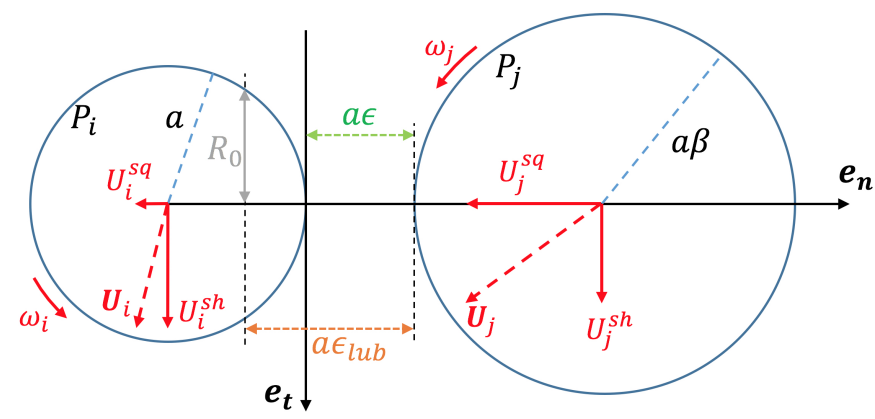

FIGURE 4 Sketch of the two interacting particles with the notations used to evaluate $\boldsymbol{F}_{i, j}^{\text {lub }}$ and $\boldsymbol{T}_{i, j}^{\text {lub }}$.

From the lubrication theory ${ }^{[17,22]}, \boldsymbol{F}_{i, j}^{\text {lub }}$ and $\boldsymbol{T}_{i, j}^{\text {lub }}$ are given by

$$
\left\{\begin{aligned}
\boldsymbol{F}_{i, j}^{\text {lub }} & =\left(\boldsymbol{F}_{i, j}^{\text {lub,sq }}+\boldsymbol{F}_{i, j}^{\text {lub,sh }}\right){ }_{\left[\epsilon_{\mathrm{col}}, \epsilon_{\mathrm{lub}}\right](\epsilon)}, \\
\boldsymbol{T}_{i, j}^{\text {lub }} & =\boldsymbol{T}_{i, j}^{\text {lub,sh }} 1_{\left[\epsilon_{\mathrm{col}}, \epsilon_{\mathrm{lub}}\right](\epsilon)},
\end{aligned}\right.
$$


with

$$
\left\{\begin{aligned}
\frac{F_{i, j}^{\mathrm{lub}, \mathrm{sq}}}{\pi \mu a U_{i, j}^{s q}} & =-\frac{6 R_{0}^{4}}{4 H_{0}^{2}} \frac{1}{\epsilon} e_{n}, \\
\frac{F_{i, j}^{\mathrm{lub}, \mathrm{sh}}}{\pi \mu a U_{i, j}^{\mathrm{sh}}} & =\int_{0}^{R_{0}}\left[-P_{0} R+\partial_{Z} V_{0}-\partial_{Z} U_{0}\right] R \mathrm{~d} R \boldsymbol{e}_{\boldsymbol{t}}, \\
\frac{T_{i, j}^{\mathrm{lub}, \mathrm{sh}}}{\pi \mu a^{2} U_{i, j}^{\mathrm{sh}}} & =\int_{0}^{R_{0}}\left[\partial_{Z} U_{0}-\partial_{Z} V_{0}\right] R \mathrm{~d} R e_{n} \times e_{\boldsymbol{t}},
\end{aligned}\right.
$$

and

$$
\left\{\begin{aligned}
U_{i, j}^{\mathrm{sq}} & =U_{i}^{\mathrm{sq}}-U_{j}^{\mathrm{sq}}, \\
U_{i, j}^{\mathrm{sh}} & =U_{i}^{\mathrm{sh}}-U_{j}^{\mathrm{sh}}+\left(\omega_{i}-\omega_{j} \beta\right) a \\
H_{0} & =1+\frac{1}{2} a \alpha R_{0}^{2} \\
\alpha & =\frac{\beta+1}{a \beta} \\
R_{0} & =\frac{1}{\sqrt{\epsilon}} \sqrt{1-\left(1+\epsilon-\epsilon_{\mathrm{lub}}\right)^{2}}
\end{aligned}\right.
$$

The projected relative velocities of the two particles on the directions $e_{n}$ and $e_{t}$ are $U_{i, j}^{\text {sq }}$ and $U_{i, j}^{\text {sh }}$ (see Fig. 4). The indicator function $1_{\left[\epsilon_{\mathrm{lub}}, \epsilon_{\mathrm{col}}\right]}(\epsilon)$ is non-zero and equal to 1 only if $\epsilon_{\mathrm{col}} \leq \epsilon \leq \epsilon_{\mathrm{lub}}$. Hence the lubrication model is switched off when the hydrodynamics is fully resolved $\left(\epsilon \geq \epsilon_{\text {lub }}\right.$ ), and when the gap disappears ( $\epsilon \leq \epsilon_{\text {col }}$ ).

The analytic expressions of $\boldsymbol{F}_{i, j}^{\text {lub,sh }}$ and $\boldsymbol{T}_{i, j}^{\text {lub,sh }}$ are not as simple as the expression of $\boldsymbol{F}_{i, j}^{\text {lub,sq }}$. Therefore, we choose to compute the shearing components by a numerical integration. Furthermore, numerical integration will be necessary if we want to add lower orders of the lubrication forces and torques. The force $\boldsymbol{F}_{i, j}^{\text {lub,sh }}$ and the torque $\boldsymbol{T}_{i, j}^{\text {lub,sh }}$ are integrated on $n_{\text {lub }}$ sub-sets of the lubrication region $\left[0, R_{0}\right]$ as follows

$$
\begin{aligned}
\frac{\boldsymbol{F}_{i, j}^{\text {lubss }}}{\pi \mu a U_{i, j}^{\text {sq }}} & =\sum_{k=1}^{n_{\text {lub }}} \int_{\boldsymbol{R}_{k}}\left[-P_{0} R+\partial_{Z} V_{0}-\partial_{Z} U_{0}\right] R \mathrm{~d} R \boldsymbol{e}_{\boldsymbol{t}}, \\
\frac{\boldsymbol{T}_{i, j}^{\text {lub,sh }}}{\pi \mu a^{2} U_{i, j}^{\text {sh }}} & =\sum_{k=1}^{n_{\text {lub }}} \int_{\boldsymbol{R}_{k}}\left[\partial_{Z} U_{0}-\partial_{Z} V_{0}\right] R \mathrm{~d} R \boldsymbol{e}_{\boldsymbol{n}} \times \boldsymbol{e}_{\boldsymbol{t}},
\end{aligned}
$$

where $\boldsymbol{R}_{k}=\left[\frac{k-1}{n_{\mathrm{lub}}} R_{0}, \frac{k}{n_{\mathrm{lub}}} R_{0}\right]$

The local lubrication correction model (LLCM) is then built such that the total hydrodynamic force and torque acting on $P_{i}$ are approximated by

$$
\left\{\begin{array}{l}
\boldsymbol{F}_{i}^{\mathrm{hyd}} \approx \boldsymbol{F}_{i}^{\mathrm{solv}}+\boldsymbol{F}_{i}^{\mathrm{lub}}, \\
\boldsymbol{T}_{i}^{\mathrm{hyd}} \approx \boldsymbol{T}_{i}^{\text {solv }}+\boldsymbol{T}_{i}^{\text {lub }} .
\end{array}\right.
$$

As the lubrication corrections $\boldsymbol{F}_{i, j}^{\mathrm{lub}}$ and $\boldsymbol{T}_{i, j}^{\mathrm{lub}}$ are the dominant orders of the hydrodynamics acting on the inner 
region of $P_{i}$, when the flow in the gap is in the Stokes regime, $\boldsymbol{F}_{i}^{\text {lub }}$ and $\boldsymbol{T}_{i}^{\text {lub }}$ are not identical to the degraded hydrodynamics $\boldsymbol{F}_{i}^{\mathrm{deg}}$ and $\boldsymbol{T}_{i}^{\mathrm{deg}}$. Nevertheless, we have shown ${ }^{[17]}$ that the approximation $\boldsymbol{F}_{i}^{\mathrm{deg}} \approx \boldsymbol{F}_{i}^{\text {lub }}$ can be made as long as the gap Reynolds numbers

$$
R e_{i, j}^{\text {gap }}=\frac{\rho a_{i} \epsilon\left\|U_{i}-U_{j}\right\|}{\mu}
$$

remains small for all $P_{j}$ in interaction with $P_{i}\left(a_{i}\right.$ the radius of $\left.P_{i}\right)$, during the simulations. In particular, the Reynolds number $\mathrm{Re}^{\text {lub }}$ needs to be moderate (typically $\operatorname{Re}^{\mathrm{lub}}<10^{3}$ ):

$$
\operatorname{Re}^{\mathrm{lub}}=\frac{\rho \epsilon_{\mathrm{lub}} Q^{\max }}{\mu}
$$

with $Q^{\max }=\max _{t}\left(\max _{(i, j) \in \llbracket 1, N \rrbracket^{2}}\left(a_{i}\left\|\boldsymbol{U}_{\boldsymbol{i}}(t)-\boldsymbol{U}_{j}(t)\right\|\right)\right)$.

This constraint also underlines the limitation of the LLCM to moderate Reynolds number flows. Indeed, inertia effects of the fluid in the gap are not corrected by the LLCM. Another limitation of the LLCM concerns the many-body interactions, which refer to the hydrodynamics action on a particle generated by nearby particles in interaction. As the lubrication corrections are made in an additive pairwise-fashion (see Eq. (16)), the only many-body interactions, present in the simulated flow, are the ones resolved by the numerical method (included in $\boldsymbol{F}_{i}^{\text {solv }}$ ).

For ellipsoidal particles, the lubrication corrections are evaluated on the virtual spheres introduced in Sec. 3.2. The approximation of the particle surfaces by the spheres is an additional source of uncertainty from the LLCM. Therefore, the size of the grid elements might need to be reduced for ellipsoidal particles with extreme aspect ratios to limit the lubrication area to contact point neighborhoods where the virtual spheres fit the most the particle surfaces.

\section{5 | The soft-sphere collision model.}

The collision model chosen here is based on the soft-sphere approach used by Costa et al. ${ }^{[26,31,32]}$. The deformation of particles during contact is modeled by the overlap between a particle and an obstacle (particle or wall). From the overlap measurement, normal and tangential contact forces are computed using a mass-spring-dashpot system and a Coulomb-type threshold for the tangential component. For a given particle $P_{i}$, collision forces $\boldsymbol{F}_{i}^{\text {coll }}$ and torque $\boldsymbol{T}_{i}^{\text {coll }}$ are decomposed as

$$
\left\{\begin{array}{l}
\boldsymbol{F}_{i}^{\text {coll }}=\sum_{j \neq i} \boldsymbol{F}_{i, j}+\boldsymbol{F}_{i, \text { wall }}, \\
\boldsymbol{T}_{i}^{\text {coll }}=\sum_{j \neq i} \boldsymbol{T}_{i, j}+\boldsymbol{T}_{i, \text { wall }},
\end{array}\right.
$$

where $\boldsymbol{F}_{i, j}$ is the collision force of the interacting particles $P_{i}$ and $P_{j}, \boldsymbol{F}_{i, \text { wall }}$ is the collision force of $P_{i}$ with a wall. $\boldsymbol{T}_{i, j}$ and $\boldsymbol{T}_{i, \text { wall }}$ are the corresponding collision torques. The force and the torque on $P_{i}$ resulting from the particle-particle interactions between $P_{i}$ and $P_{j}$ are defined using a local system of coordinates $\left(\boldsymbol{e}_{\boldsymbol{n}}, \boldsymbol{e}_{\boldsymbol{t}}\right)$ (Fig. 5):

$$
\left\{\begin{array}{l}
\boldsymbol{F}_{i, j}=\boldsymbol{F}_{n}+\boldsymbol{F}_{t}, \\
\boldsymbol{T}_{i, j}=\boldsymbol{e}_{\boldsymbol{n}} \times\left(\boldsymbol{F}_{t}\right),
\end{array}\right.
$$


with

$$
\left\{\begin{array}{l}
\boldsymbol{F}_{n}=-\boldsymbol{\delta}_{\boldsymbol{n}} k_{\mathrm{n}}-\gamma_{\mathrm{n}}\left(\boldsymbol{U}_{i, j} \cdot \boldsymbol{e}_{\boldsymbol{n}}\right) \boldsymbol{e}_{\boldsymbol{n}}, \\
\boldsymbol{F}_{t}=\min \left(\left\|-\boldsymbol{\delta}_{\boldsymbol{t}} k_{\mathrm{t}}-\gamma_{\mathrm{t}}\left(\boldsymbol{U}_{i, j} \cdot \boldsymbol{e}_{t}\right) \boldsymbol{e}_{t}\right\|,\left\|\mu_{c} \boldsymbol{F}_{n}\right\|\right) \boldsymbol{e}_{t},
\end{array}\right.
$$

where $a$ is the radius of $P_{i}, \boldsymbol{\delta}_{\boldsymbol{n}}$ (respectively, $\boldsymbol{\delta}_{\boldsymbol{t}}$ ) is the normal (respectively, tangential) overlap, $k_{\mathrm{n}}$ (respectively, $k_{\mathrm{t}}$ ) is the normal (respectively, tangential) stiffness, $\mu_{c}$ is the coefficient of sliding friction, and $\gamma_{n}$ (respectively, $\gamma_{t}$ ) is the normal (respectively, tangential) damping coefficient of the spring-dashpot model. The relative velocity of the two particles $U_{i, j}$ at the contact point is given by $U_{i, j}=U_{i}+a \omega_{i} \times e_{n}-\left(U_{j}-a \beta \omega_{j} \times e_{n}\right)$.

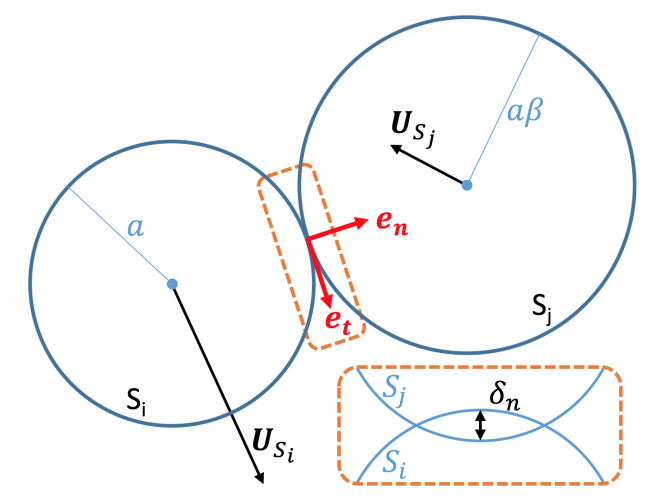

FIGURE 5 Contact of two particles with notations associated to the soft sphere model.

The normal overlap distance $\boldsymbol{\delta}_{\boldsymbol{n}}$ is given by

$$
\boldsymbol{\delta}_{\boldsymbol{n}}=\max \left(0, a(1+\beta)+\epsilon_{\mathrm{col}}(a+\beta a)-\left\|\boldsymbol{X}_{i}-\boldsymbol{X}_{j}\right\|\right) \boldsymbol{e}_{\boldsymbol{n}},
$$

where $\boldsymbol{e}_{\boldsymbol{n}}=\frac{\boldsymbol{X}_{i}-\boldsymbol{X}_{j}}{\left\|\boldsymbol{X}_{i}-\boldsymbol{X}_{j}\right\|}$ as shown Fig. 5. The tangential overlap distance $\boldsymbol{\delta}_{\boldsymbol{t}}$ is obtained by integrating the relative tangential velocity at the point of contact while the Coulomb's law is verified. Therefore, the tangential overlap distance $\delta_{t}{ }^{n+1}$ at the time step $n+1$ is obtained by

$$
\delta_{t}^{n+1}=\frac{1}{k_{\mathrm{t}}}\left(-\mu_{c}\left\|\boldsymbol{F}_{n}\right\| e_{t}-\gamma_{\mathrm{t}}\left(\boldsymbol{U}_{i, j} \cdot \boldsymbol{e}_{\boldsymbol{t}}\right) \boldsymbol{e}_{\boldsymbol{t}}\right)
$$

when the particle is sliding (i.e. $\left\|\boldsymbol{F}_{t}\right\|>\mu_{\mathrm{c}}\left\|\boldsymbol{F}_{n}\right\|$ ), and by

$$
\delta_{t}^{n+1}=R_{\delta_{t}} \cdot \delta_{t}^{n}+\int_{t^{n}}^{t^{n+1}}\left(U_{i, j} \cdot e_{t}\right) e_{t} \mathrm{~d} t
$$

when the particle is sticking to the obstacle (i.e. $\left.\left\|\boldsymbol{F}_{t}\right\| \leq \mu_{\mathrm{c}}\left\|\boldsymbol{F}_{n}\right\|\right)$. The rotation tensor $R_{\delta_{t}}$ moves $\boldsymbol{\delta}_{\boldsymbol{t}}{ }^{n}$ to the new local coordinate system at the state $n+1$, and

$$
\boldsymbol{e}_{\boldsymbol{t}}=\frac{-\boldsymbol{\delta}_{\boldsymbol{t}} k_{\mathrm{t}}-\gamma_{\mathrm{t}}\left(\boldsymbol{U}_{i, j}-\left(\boldsymbol{U}_{i, j} \cdot \boldsymbol{e}_{\boldsymbol{n}}\right) \boldsymbol{e}_{\boldsymbol{n}}\right)}{\left\|-\boldsymbol{\delta}_{\boldsymbol{t}} k_{\mathrm{t}}-\gamma_{\mathrm{t}}\left(\boldsymbol{U}_{i, j}-\left(\boldsymbol{U}_{i, j} \cdot \boldsymbol{e}_{\boldsymbol{n}}\right) \boldsymbol{e}_{\boldsymbol{n}}\right)\right\|} .
$$


The parameters of the spring-dashpot model $\gamma_{\mathrm{n}}, \gamma_{\mathrm{t}}, k_{\mathrm{n}}$, and $k_{\mathrm{t}}$ are calculated from the coefficient of normal (respectively, tangent) restitution $\xi_{\max , \mathrm{n}}$ (respectively, $\xi_{\max , \mathrm{t}}$ ) of dry collision and the contact time $\tau_{\mathrm{c}}$, as follows:

$$
\left\{\begin{array}{l}
k_{\mathrm{n}}=\frac{m^{*}\left(\pi^{2}+\ln ^{2}\left(\xi_{\max , \mathrm{n}}\right)\right)}{\tau_{\mathrm{c}}^{2}}, \\
\gamma_{\mathrm{n}}=-\frac{2 m^{*} \ln \left(\xi_{\max , \mathrm{n}}\right)}{\tau_{\mathrm{c}}}, \\
k_{\mathrm{t}}=\frac{m_{t}^{*}\left(\pi^{2}+\ln ^{2}\left(\xi_{\max , \mathrm{t}}\right)\right)}{\tau_{\mathrm{c}}^{2}}, \\
\gamma_{\mathrm{t}}=-\frac{2 m_{\mathrm{t}}^{*} \ln \left(\xi_{\max , \mathrm{t}}\right)}{\tau_{\mathrm{c}}},
\end{array}\right.
$$

with the effective mass

$$
m^{*}=\frac{m_{i} m_{j}}{m_{i}+m_{j}},
$$

and

$$
m_{\mathrm{t}}^{*}=m^{*} \frac{K^{2}}{K^{2}+1},
$$

with $K^{2}=2 / 5$ the normalized radius of gyration for spherical particles.

The characteristics of the elastic properties of the particles are $\xi_{\max , n}, \xi_{\max , \mathrm{t}}$, and $\tau_{\mathrm{c}}$. As noticed by lzard et al. ${ }^{\text {[23] }}$, the relation between $\tau_{\mathrm{c}}$ and $k_{\mathrm{n}}$ is unusual, but several studies show that the normal stiffness can be underestimated without modification of the dynamics of a dry system. Such an assumption allows to reduce the simulation time since the collision characteristic time will be larger than the particle characteristic deformation time.

To ensure the stability of the model and the conservation of the momentum, Costa et al. ${ }^{[26]}$ advised that the time step of the overall numerical algorithm $\Delta t$ has to be chosen as a multiple of the contact time $\tau_{\mathrm{c}}$ (at least during the collision). This condition guarantees a zero overlap at the end of the collision and allows the fluid to adapt itself to sudden changes in velocity of the colliding particles.

The force $\boldsymbol{F}_{i, \text { wall }}$ and the torque $\boldsymbol{T}_{i, \text { wall }}$ are assumed to be equivalent to the asymptotic case $\beta \rightarrow+\infty$ and $m_{P_{j}} \rightarrow+\infty$.

For non-spherical particles, the ellipsoids are approximated as spherical particles with the same mass as the whole particles and with a radius corresponding to the local Gaussian curvature at the contact point ${ }^{[15][33]}$. During collisions, the radii of the approximating spheres remain constant simplifying the problem to the collision between two unequal spheres. The centers of the approximating colliding spheres are stored at the time step before the gap width becomes negative (particles overlap) and updated during the collision using the particle velocity and the rotation matrix introduced above. These constraints prevent potential non-converged computations of the virtual spheres to destabilize the collision model.

\subsection{Numerical resolution of the particle dynamics.}

Among the forces acting on the particle, the short-range hydrodynamics (lubrication) and collision forces have time scales smaller than the time scale associated with fluid flow. The particle dynamics is therefore computed at a smaller time step $\delta t=\frac{\Delta t}{n_{t}}$, with $n_{t}$ arbitrary chosen large, to accurately integrate (in time) short-range interaction forces. Numerical simulations have shown that changing $n_{t}$ between $10^{2}$ and $10^{3}$ does not affect significantly the results. 
Since the motion of the particles occurs at a time step smaller than $\Delta t$, it is not necessary to re-compute the resolved hydrodynamic forces $\boldsymbol{F}_{i}^{\text {solv }}$ at each sub-time step $\delta t$. Hence, particle dynamics is solved at each sub-time step $\delta t$ with updated short-range interactions and "frozen" resolved hydrodynamics.

The Newton Euler equations of conservation (2) and (3) are discretized using a semi-implicit Euler scheme as follows:

$$
\begin{aligned}
\boldsymbol{U}_{i}^{m+1}= & \boldsymbol{U}_{i}^{m}+\frac{\delta t}{m_{i}}\left[\boldsymbol{F}_{i}^{\mathrm{solv}}\right]^{n+1} \\
& +\frac{\delta t}{m_{i}}\left[\boldsymbol{F}_{i}^{\mathrm{coll}}+\boldsymbol{F}_{i}^{\mathrm{ext}}+\boldsymbol{F}_{i}^{\mathrm{lub}}\right]^{m} . \\
\boldsymbol{\Omega}_{i}^{m+1}= & \left(J_{i}^{m+1}\right)^{-1}\left(J_{i}^{m} \boldsymbol{\Omega}_{i}^{m}\right. \\
& \left.+\delta t\left(\left[\boldsymbol{T}_{i}^{\mathrm{solv}}\right]^{n+1}+\left[\boldsymbol{T}_{i}^{\mathrm{coll}}+\boldsymbol{T}_{i}^{\text {lub }}\right]^{m}\right)\right),
\end{aligned}
$$

where $m$ denotes the "sub-state" of the system at the time $t=t_{n}+m \delta t$ with $t_{n}$ the time at the state $n$ of the system. Since the particle dynamics are solved after the correction step but before the penalization, the force $\left[\boldsymbol{F}_{i}^{\text {solv }}\right]^{n+1}$ and torques $\left[\boldsymbol{T}_{i}^{\text {solv }}\right]^{n+1}$ are computed from the fields $\left(p^{n+1}, \tilde{\boldsymbol{u}}\right)$, and not $\left(p^{n+1}, \boldsymbol{u}^{n+1}\right)$.

Position and orientation are then integrated implicitly as follows:

$$
\left\{\begin{array}{l}
\boldsymbol{X}_{i}^{m+1}=\boldsymbol{X}_{i}^{m}+\delta t \boldsymbol{U}_{i}^{m+1} \\
\boldsymbol{\Theta}_{i}^{m+1}=\boldsymbol{\Theta}_{i}^{m}+\delta t \boldsymbol{\Omega}_{i}^{m+1}
\end{array}\right.
$$

Higher order integration schemes than the semi-implicit Euler scheme would be preferable for practical industrial applications where accuracy and efficiency are critical. Nevertheless, the Euler scheme provides a simple numerical framework to test the lubrication models which are the focus of our works.

\subsection{1 | Numerical stability: time step adaptation.}

To ensure the stability of the whole numerical method, the time step $\Delta t$ is adapted such that the Courant - Friedrichs - Lewy condition is satisfied: $\Delta t=\beta_{\tau} \Delta x / V_{\max }$ where $\beta_{\tau} \leq 1$ is chosen arbitrarily, $\Delta x$ is the characteristic length of the grid cells and $V_{\max }$ is the maximum of the velocity absolute value computed on the grid cells.

When particles are near contact, the time step $\Delta t$ has to satisfy the stability condition of the collision model (see Sec. 3.5). Therefore, when lubrication corrections are active (i.e. collision might occur) $\Delta t$ is chosen such that $\tau_{\mathrm{c}}=N_{t} \Delta t$ (with $\tau_{\mathrm{c}}$ the contact time) and $\Delta t \leq \beta_{\tau} \frac{\Delta x}{V_{\max }}$, where $N_{t}>0$ an integer ( $N_{t}=8$, if not explicitly stated otherwise).

\section{4 | NUMERICAL SIMULATIONS.}

As long as ellipsoidal particles remain far away from each other, accurate numerical simulations of particle-laden flows can be run without introducing additional models. Hence, we will focus in this section on evaluating the accuracy of lubrication models, as unresolved hydrodynamics between bodies in close interaction are critical to simulate suspension and are often overlooked for non-spherical particles. Even if the numerical method described above can handle any kind of particle geometry as long as the surfaces of interacting particles are convex around their contact points, we choose to limit our validation to ellipsoidal particles for the sake of simplicity. 
After checking that the numerical method can accurately simulate the motion of an isolated ellipsoid in a sheared flow, the LLCM is tested in depth on a prolate particle moving toward a planar wall.

\section{1 | Single particle in a sheared flow.}

Before looking at the lubrication correction, the numerical method has been tested with an isolated spheroidal particle in a simple shear flow. Non-slip boundary conditions are assumed on the top and bottom faces of the domain while periodic boundary conditions are considered on the all others faces. The neutrally buoyant particle is place in the middle of the domain with $\Theta_{z}=\Theta_{y}=0$, and is initially at rest.

The analytical solution for the angular velocity $\Omega_{z}$ is given ${ }^{[34]}$ in the inertialess regime $\left(\operatorname{Re}_{e q}=0\right)$ by

$$
\Omega_{z}=-\frac{\dot{\gamma}}{a^{2}+b^{2}}\left(b^{2} \cos ^{2}\left(\Theta_{z}\right)+a^{2} \sin ^{2}\left(\Theta_{z}\right)\right)
$$

where $\dot{\gamma}$ is the imposed shear rate, and the semi axes $a$ and $b$ are the polar and the equatorial radius of the spheroid (Fig. 2).

Simulations are performed with two spheroids with aspect ratios $\mathcal{A} \mathcal{R}=2$ and $\mathcal{A} \mathcal{R}=1 / 3$. Horizontal velocity of the top and bottom walls are set such that the Reynolds number is

$$
\operatorname{Re}_{\mathrm{eq}}=\frac{\rho \dot{\gamma} D_{e q}^{2}}{\mu}=0.1 \text {, }
$$

where $D_{\text {eq }}$ is the particle equivalent diameter. The computational domain of size $\left[10 D_{\text {eq }}\right]^{3}$ is mapped with a uniform Cartesian mesh. No-slip boundary conditions are assumed on the particle and wall surfaces. Figure 6 shows that the simulation results, obtained with a grid spacing of $\Delta x=\Delta y=\Delta z=D_{\mathrm{eq}} / 30$, are in good agreement with the analytical solutions Eq. (33). The particle tumbling period around the spanwise axis is given by

$$
T=\frac{2 \pi}{\dot{\gamma}}\left(\mathcal{A} \mathcal{R}+\frac{1}{\mathcal{A} \mathcal{R}}\right) .
$$




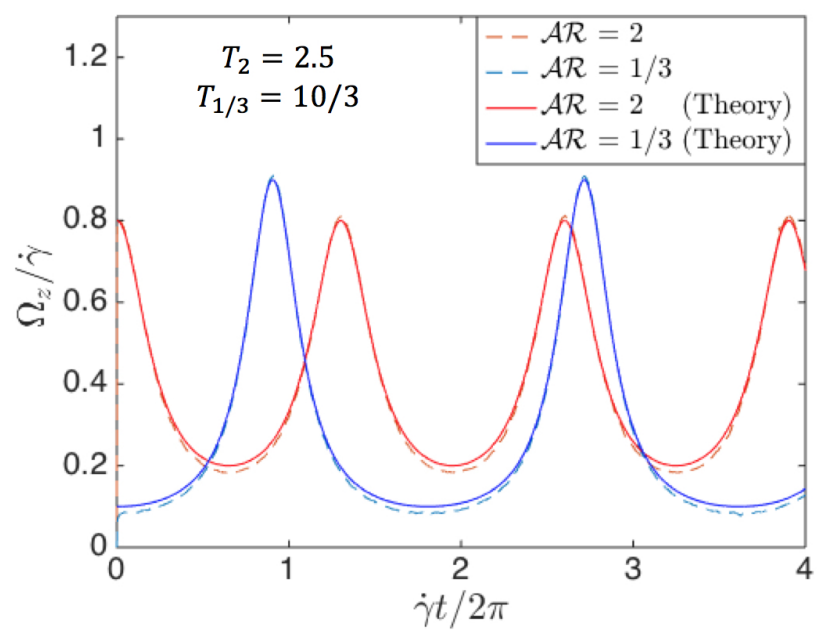

FIGURE 6 Evolution of the normalized rotation velocity $\Omega_{z}$ of a prolate (red) and oblate (blue) in a shear flow. Simulation results (dashed lines), using $h=1 / 30$ grid resolution, are compared to Jeffrey et al. solutions ${ }^{[34]}$.

\section{2 | Sedimentation toward a planar wall.}

A single particle is immersed in a domain $\left[2 D_{\text {eq }}\right]^{3}$, with $D_{\text {eq }}$ the particle equivalent diameter, uniformly meshed with cubic elements of size $\Delta x=\Delta y=\Delta z=h d$. No-slip boundary conditions are imposed on every face of the domain. The fluid is initially at rest and the particle is dropped without initial velocity such that the gap size from the top wall is given by $d_{\text {init }}$ as shown Fig. 7. The gravity field $g$ acts on the $y$-direction.

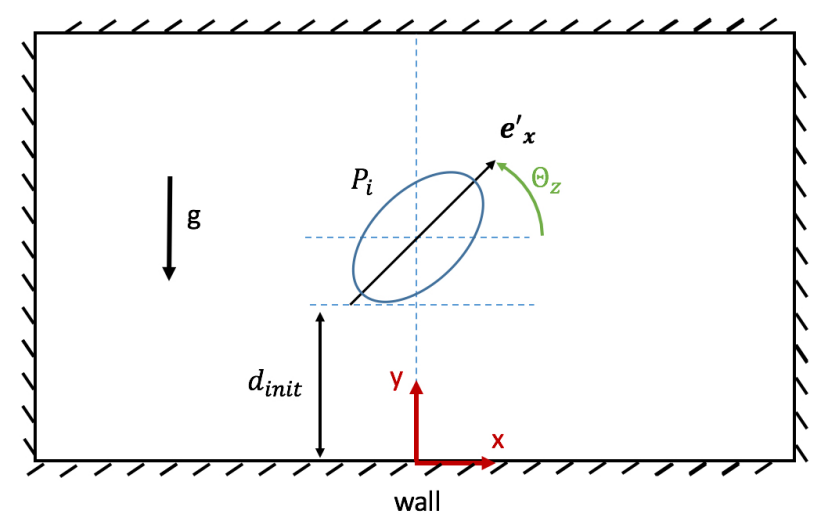

FIGURE 7 Sketch of the simulation domain with the particle initial position.

If not explicitly stated otherwise, all simulations were performed using the particle and fluid properties contained in Tab. 1. The Reynolds number

$$
\operatorname{Re}_{\mathrm{eq}}=\frac{\rho U_{\mathrm{t}} D_{\mathrm{eq}}}{\mu} \in[20,40]
$$


and Stokes number

$$
\mathrm{St}_{\mathrm{eq}}=\frac{\rho_{\mathrm{p}} U_{\mathrm{t}} D_{\mathrm{eq}}}{9 \mu} \in[16,36]
$$

are a posteriori estimated with $U_{t}$ the particle impact velocity, for all considered particle aspect ratios. At very low Reynolds and Stokes number ( $\mathrm{Re}_{\mathrm{eq}} \ll 10$ and $\mathrm{St}$ eq $\ll 10$, typically) the particle settling above the wall can be qualitatively achieved by any lubrication model, and might even be done without corrections. Due to the lack of measurements and the high cost of DNS simulation of the whole particle trajectory, the accuracy of the lubrication models will be not quantitatively assess in this fully viscous regime. We choose to focus our investigation on the transition regime between the Stokes and fully inertial regime as performance differences between lubrication models are easier to highlight.

DNS simulations are performed using $h=1 / 200$ and the particle trajectories are only computed for gap sizes ranging from $d_{\text {init }}$ to $d=2.5 \mathrm{~mm}$. Hence, DNS simulations can be considered as references with at least five grid elements in the gap at all times (i.e. no collision or lubrication corrections are made).

\begin{tabular}{|lccc}
\hline Fluid density & $\rho$ & 1000 & $\mathrm{~kg} \cdot \mathrm{m}^{-3}$ \\
\hline Fluid dynamic viscosity & $\mu$ & 1 & $\mathrm{~Pa} \cdot \mathrm{s}$ \\
\hline Equivalent diameter & $D_{\mathrm{ep}}$ & 10 & $\mathrm{~cm}$ \\
\hline Particle density & $\rho_{\mathrm{p}}$ & 8000 & $\mathrm{~kg} \cdot \mathrm{m}^{-3}$ \\
\hline Initial position & $d_{\text {init }}$ & 1.25 & $\mathrm{~cm}$ \\
\hline Initial orientation & $\Theta_{z, \text { init }}$ & 0 & $\mathrm{rad}$ \\
\hline Particle aspect ratio & $\mathcal{A R}$ & 2 & \\
\hline Galileo number & $\mathrm{Ga}$ & 262 & \\
\hline
\end{tabular}

TAB LE 1 Particle and fluid properties.

Numerical experiments have shown that the number of particle surface mesh elements (starting from about three thousand elements) has a limited impact on the solution, for all particle aspect ratio considered. Therefore, all simulations have been performed using $N_{\mathrm{p}}=7200$.

\subsection{1 | Grid sensitivity.}

In order to estimate the rate of convergence of the numerical method, five simulations using background grid resolutions $h \in[1 / 120,1 / 40]$ are compared to a DNS solution ( $h=1 / 200$, without lubrication corrections). For all simulations, the LLCM parameter is $\epsilon_{\mathrm{lub}}=0.05$ and a constant arbitrary time step $\Delta t=2.5 \cdot 10^{-5} \mathrm{~s}$ is used for all simulations including the DNS.

Figure 8 compares the evolution of the total hydrodynamic force obtained with the LLCM to the DNS results. Strong oscillations (noises) observed on coarse meshes are due to high $\Delta x / \Delta t$ ratios $^{[35]}$. When the particle enters the lubrication area, hydrodynamic forces drop compared to DNS results. This difference is due to the lubrication model. As the minimal gap in Fig. 8 is still large $(\varepsilon=0.033)$, results could be improved by correcting lower order in $\epsilon$ of the theoretical lubrication force ${ }^{[22]} F^{\text {lub }}$. This could also explain the apparent underestimation of the hydrodynamics compared to the DNS solution when the LLCM is active (as seen in Fig. 8), since the hydrodynamic forces acting around the contact point are substituted by lubrication corrections where lubrication forces are not yet predominant. The 
observed behavior of the LLCM is likely transitional but DNS at higher resolutions would be needed to confirm it.

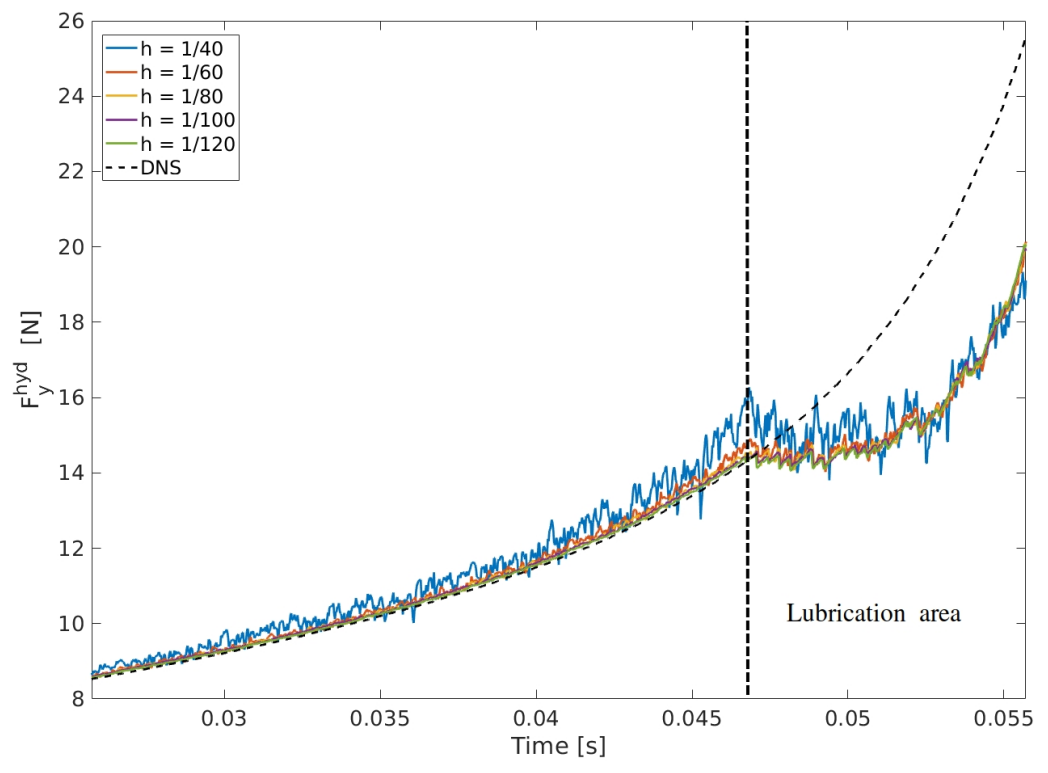

FIGURE 8 Evolution of the total hydrodynamic force, according to the $y$-direction during the approach phase. For all curves, the lubrication corrections are activated in the lubrication area $\left(\varepsilon \leq \epsilon_{\text {lub }}\right)$.

The rate of convergence on velocity decreases as the particle aspect ratio increases. Indeed, for spherical particles the rate of convergence is 0.83 while this rate is equal to 0.58 and 0.41 for particle aspect ratios $A R=1.5$ and $A R=2$, respectively. This trend can be explained by the fact that the errors due to the approximation of the particle surface at the contact point by the surface of a virtual sphere increase when the aspect ratio increases (see Sec. 4.2.3). As underlined in our previous paper ${ }^{[17]}$, the lubrication parameter $\epsilon_{\text {lub }}$ is originally defined in the LLCM as a function of the grid spacing. For instance, the numerical velocities obtained with $\epsilon_{\text {lub }}=2 / h$ should have a higher rate of convergence than results obtained with $\epsilon_{\text {lub }}=0.05$ fixed, since the solutions rely more (as $h$ decreases) on the numerical method without lubrication correction, which is second-order accurate.

\subsubsection{Sensitivity to the LLCM parameter $\epsilon_{\text {lub }}$.}

The LLCM is a model using a single parameter $\epsilon_{\text {lub }}$ which sets the minimal gap length below which lubrication corrections are needed. Several simulations have been made for different $\epsilon_{\text {lub }}$ with a grid resolution set as $h=1 / 40$. Figure 9 represents the evolution of the total hydrodynamic force acting on an $\mathcal{A} \mathcal{R}=2$ particle. 


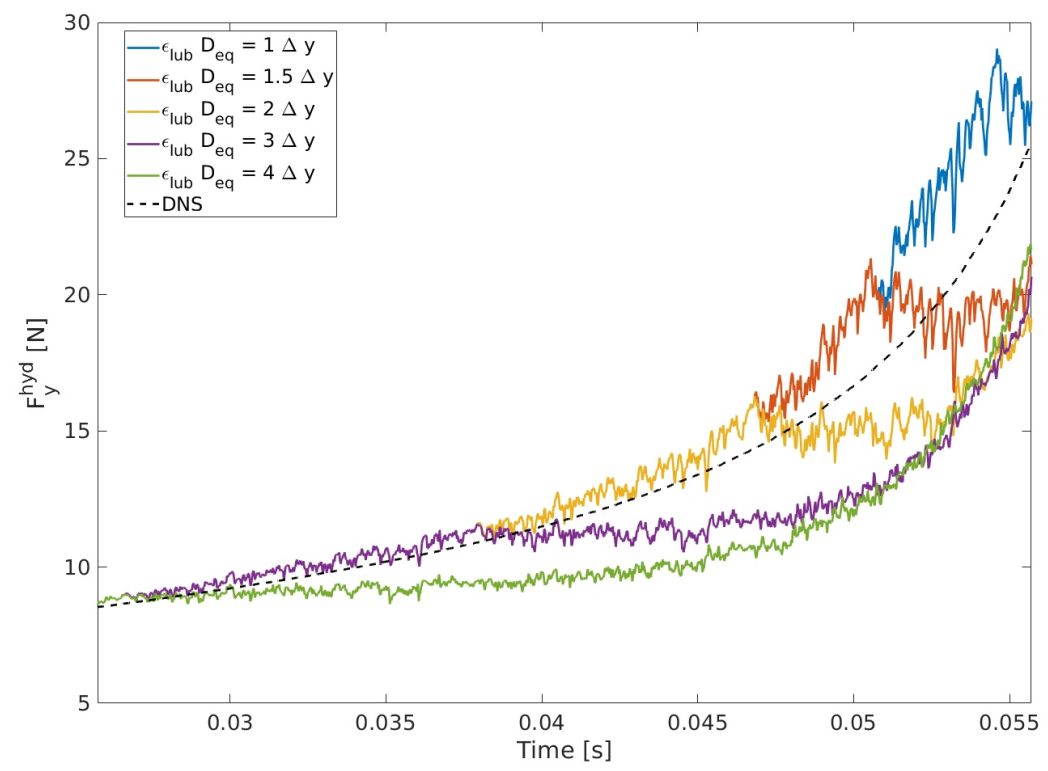

FIGURE 9 Evolution of the total hydrodynamic force, according to the $y$-direction during the approach phase for the critical lubrication distance $D_{\mathrm{eq}} \epsilon_{\text {lub }}$ equal to $3 \Delta y, 2 \Delta y, 1.5 \Delta y, 4 \Delta y$ and $1 \Delta y$ (by increasing value of $F^{\text {hyd }}$ at the last time). The grid resolution is $h=1 / 40$ and $\mathcal{A} \mathcal{R}=2$ for all curves.

For spherical particles, the lubrication parameter $\epsilon_{\text {lub }} \in[2 / h, 3 / h]$ gives accurate corrections ${ }^{[17]}$ as long as the Reynolds number in the gap is small ( $\mathrm{Re}^{\text {lub }}<10^{3}$, see Eq. 22). Similarly to the case of spherical particles, numerical simulations with $\epsilon_{\text {lub }} \in[2 / h, 3 / h]$ give hydrodynamic forces closer to the hydrodynamic force obtained by the DNS, as shown in Tab. 2. Numerical experiments have shown that $\epsilon_{\text {lub }} \in[2 / h, 3 / h]$ remains the best choice independently of the particle aspect ratio (Tab. 2) and also independently of the particle orientation (Tab. 3). Hence, no tabulation of $\epsilon_{\text {lub }}$ is needed as $\epsilon_{\text {lub }}$ is invariant with respect to particle orientation and aspect ratio.

\begin{tabular}{ccccccc}
\hline $\mathcal{A} \mathcal{R}$ & $D_{\mathrm{vr}} / D_{\text {eq }}$ & $\epsilon_{\text {lub }}=1 / h$ & $\epsilon_{\text {lub }}=1.5 / h$ & $\epsilon_{\text {lub }}=2 / h$ & $\epsilon_{\text {lub }}=3 / h$ & $\epsilon_{\text {lub }}=4 / h$ \\
\hline 1 & 1 & $+0.78 \%$ & $+0.72 \%$ & $+0.58 \%$ & $-0.53 \%$ & $-0.74 \%$ \\
1.5 & 1.32 & $+0.86 \%$ & $+0.77 \%$ & $+0.59 \%$ & $-0.66 \%$ & $-1.13 \%$ \\
2 & 1.58 & $+1.02 \%$ & $+0.91 \%$ & $+0.70 \%$ & $-0.80 \%$ & $-1.35 \%$ \\
2.5 & 1.86 & $+1.05 \%$ & $+0.97 \%$ & $+0.75 \%$ & $-0.73 \%$ & $-1.08 \%$ \\
\hline
\end{tabular}

TA B LE 2 Relative L2 error compared to the DNS velocity. A positive error indicates an overestimation of the velocity. The grid resolution is $h=1 / 40$ for all cases. The average diameter of the virtual sphere when lubrication correction are applied is denoted $D_{\mathrm{vr}}$. 


\begin{tabular}{cccccc}
\hline$\Theta_{\text {z,init }}$ & $D_{\mathrm{vr}} / D_{\text {eq }}$ & $\epsilon_{\text {lub }}=1 / h$ & $\epsilon_{\text {lub }}=2 / h$ & $\epsilon_{\text {lub }}=3 / h$ & $\epsilon_{\text {lub }}=4 / h$ \\
\hline 0 & 1.58 & $+1.02 \%$ & $+0.70 \%$ & $-0.80 \%$ & $-1.35 \%$ \\
$\pi / 6$ & 0.92 & $+0.92 \%$ & $+0.81 \%$ & $-0.30 \%$ & $-0.50 \%$ \\
$\pi / 4$ & 0.64 & $+0.87 \%$ & $+0.69 \%$ & $-0.18 \%$ & $-0.39 \%$ \\
$\pi / 3$ & 0.49 & $+0.71 \%$ & $+0.58 \%$ & $-0.15 \%$ & $-0.36 \%$ \\
$\pi / 2$ & 0.4 & $+0.51 \%$ & $+0.35 \%$ & $-0.13 \%$ & $-0.23 \%$ \\
\hline
\end{tabular}

TAB LE 3 Signed relative L2 error compared to the DNS particle velocity for $\mathcal{A} \mathcal{R}=2$. A positive error indicates an overestimation of the velocity. The average diameter of the virtual sphere when lubrication correction are applied is denoted $D_{\mathrm{vr}}$.

\subsection{3 | Lubrication models comparison and influence of the aspect ratio.}

In Sec. 3.4, we recalled the lubrication correction model (CLM) based on the works of Ardekani et al. ${ }^{[15]}$. The CLM model uses the lubrication forces acting on the whole surface of the virtual sphere as corrections instead of a local correction as performed by the LLCM. Figure 10 shows the evolution of the total hydrodynamic force on a prolate particle $(\mathcal{A} \mathcal{R}=2$ ) using the CLM, LLCM, or no lubrication correction. Numerical simulations were performed using an identical numerical framework (aside from the lubrication model) with a grid spacing $h=1 / 40$.

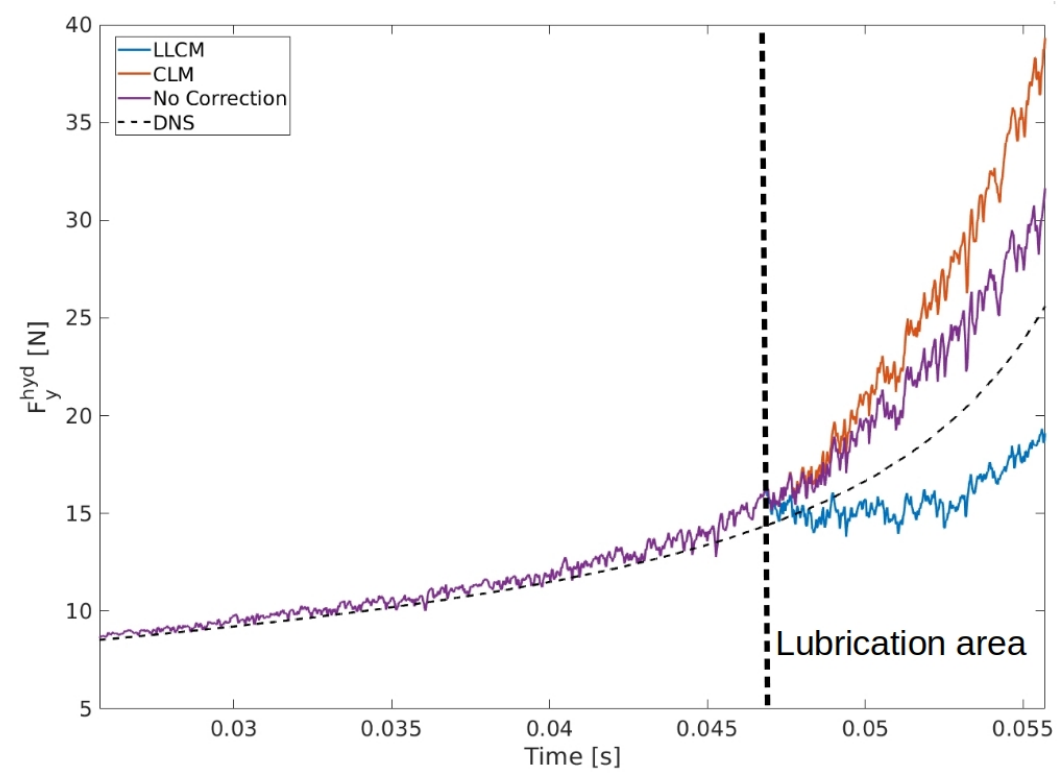

FIGURE 10 Evolution of the total hydrodynamic force acting along the $y$-direction. Simulations without lubrication correction, with LLCM and CLM are performed using $h=1 / 40$ grid resolution and $\mathcal{A} \mathcal{R}=2$. LLCM and CLM parameters are $\epsilon_{\text {lub }}=2 / h$ and $\epsilon_{\Delta x}=0.06$ (see Sec. 3.4 for details).

On the small interval $\epsilon \in[0.033,0.05]$ where the lubrication corrections are applied and a DNS solution is available (see Fig. 10), two clear behaviors of the two lubrication models are observed. Compared to the DNS, LLCM tends to 
underestimate the particle hydrodynamics while CLM overestimate these effects. Table 4 contains the relative error of simulated velocities compared to the DNS solutions for several aspect ratios. According to values in Tab. 4, there is no significant difference between velocities obtained with or without lubrication corrections which seems contradictory to results obtained in our previous works ${ }^{[17]}$ with spherical particles. However, the velocities used to evaluate the errors are obtained for $\varepsilon \geq 0.033$ which is still far from the wall. As described in further detail in Sec. 4.2.4, the hydrodynamic force typically saturates at smaller values of $\epsilon$ without lubrication corrections. This creates larger behavior differences between solution with and without lubrication which justify the use of lubrication correction models.

\begin{tabular}{cccc}
\hline $\mathcal{A R}$ & LLCM & CLM & No Correction \\
\hline 1 & $0.58 \%$ & $0.79 \%$ & $0.78 \%$ \\
1.25 & $0.50 \%$ & $0.74 \%$ & $0.72 \%$ \\
\hline 1.5 & $0.59 \%$ & $0.90 \%$ & $0.86 \%$ \\
\hline 1.75 & $0.67 \%$ & $1.04 \%$ & $0.96 \%$ \\
\hline 2 & $0.70 \%$ & $1.16 \%$ & $1.02 \%$ \\
\hline 2.5 & $0.75 \%$ & $1.22 \%$ & $1.06 \%$ \\
\hline
\end{tabular}

TA B LE 4 Relative L2 error compared to the DNS velocity for simulations using LLCM, CLM or no lubrication correction. Grid resolution is $h=1 / 40$ and $\Theta_{z \text {,init }}=0$ for all aspect ratio. LLCM and CLM parameters are $\epsilon_{\text {lub }}=2 / h$ and $\epsilon_{\Delta x}=0.06$.

Table 4 shows mainly the velocity errors increase with the particle aspect ratio for all cases at different rates. For both lubrication models, this observation underlines the influence of the approximation of the particle surface at the contact point by the surface of a virtual sphere. Similarly, the simulation accuracy decreases with the incoming angles $\Theta_{z}$ (see tab. 3). For $\Theta_{z}=\pi / 2$, the two main curvatures at the contact point are identical. Therefore, the virtual sphere surface fits the particle surface with a minimal error which reduces the errors of the lubrication corrections. The LLCM errors are the smallest for all aspect ratios and increase with the lowest rate. However, the LLCM is about $10 \%$ more expensive than the CLM, and $10-15 \%$ more expensive than without corrections. The additional cost comes from the search of the particle surface grid elements in the lubrication area. Therefore, a large $N_{p}$ leads to a higher cost of the LLCM compared to CLM. Despite the extra computational cost, the LLCM is the most reliable method for non-spherical particles as it provides higher accuracy and does not require to perform expensive tabulations.

\subsection{4 | Particle collision with a wall.}

In this section, simulations are run until the particle reaches an equilibrium. As the particle goes closer than $d=2.5 \mathrm{~mm}$ from the wall, DNS simulations of the whole trajectory are not possible (there cannot be enough grid elements in the gap to properly capture the flow around the particle). Figures 11 and 12 show the evolution of the hydrodynamic force and particle vertical velocity around the first impact with the wall. The Reynolds number $\operatorname{Re}_{\mathrm{eq}} \approx 35$ and Stokes number $\mathrm{St}_{\mathrm{eq}} \approx 31$ are estimated a posteriori with the particle maximal particle velocity (i.e. impact velocity). The Galileo number is $\mathrm{Ga}=264$.

Two motions of the particle are observed in Fig. 12. The particle is colliding with the wall for the simulations using LLCM or no lubrication corrections while it is maintained in suspension above the wall if the CLM is considered. Experimental recordings ${ }^{[22]}$ of the trajectory of an identical prolate $(\mathcal{A R}=2)$ approaching a planar wall at low Stokes 


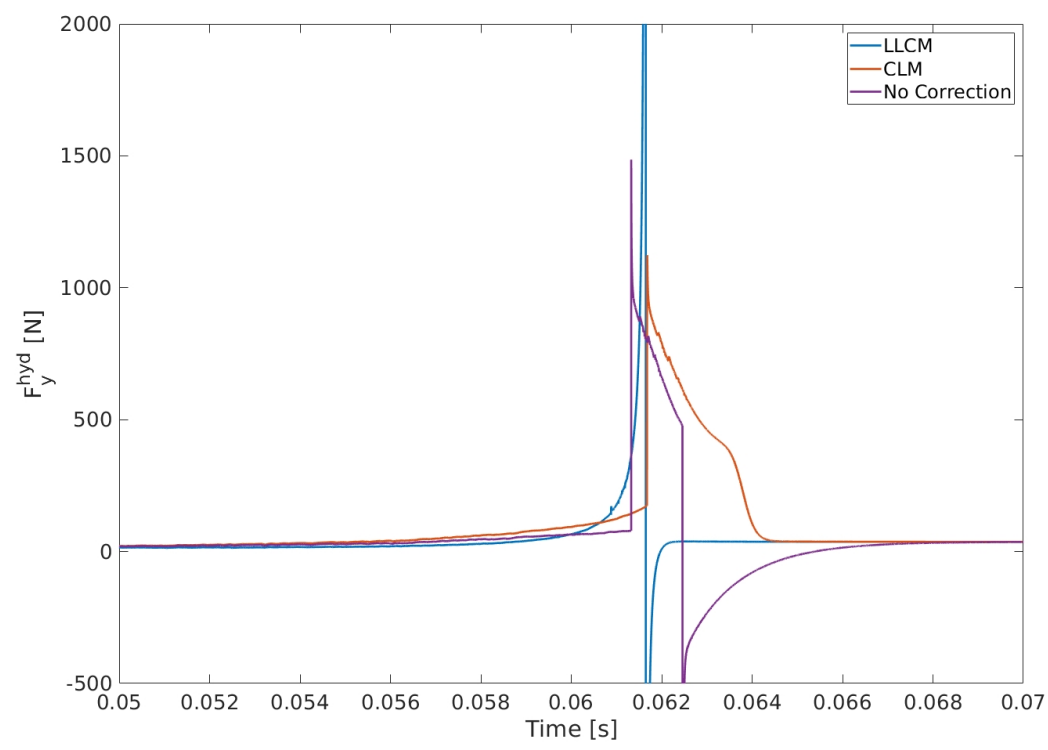

FIGURE 11 Evolution of the total hydrodynamic force acting along the $y$-direction. Simulations without lubrication correction, with LLCM and CLM are performed using $h=1 / 40$ grid resolution and $\mathcal{A} \mathcal{R}=2$. LLCM and CLM parameters are $\epsilon_{\text {lub }}=2 / h$ and $\epsilon_{\Delta x}=0.06$.

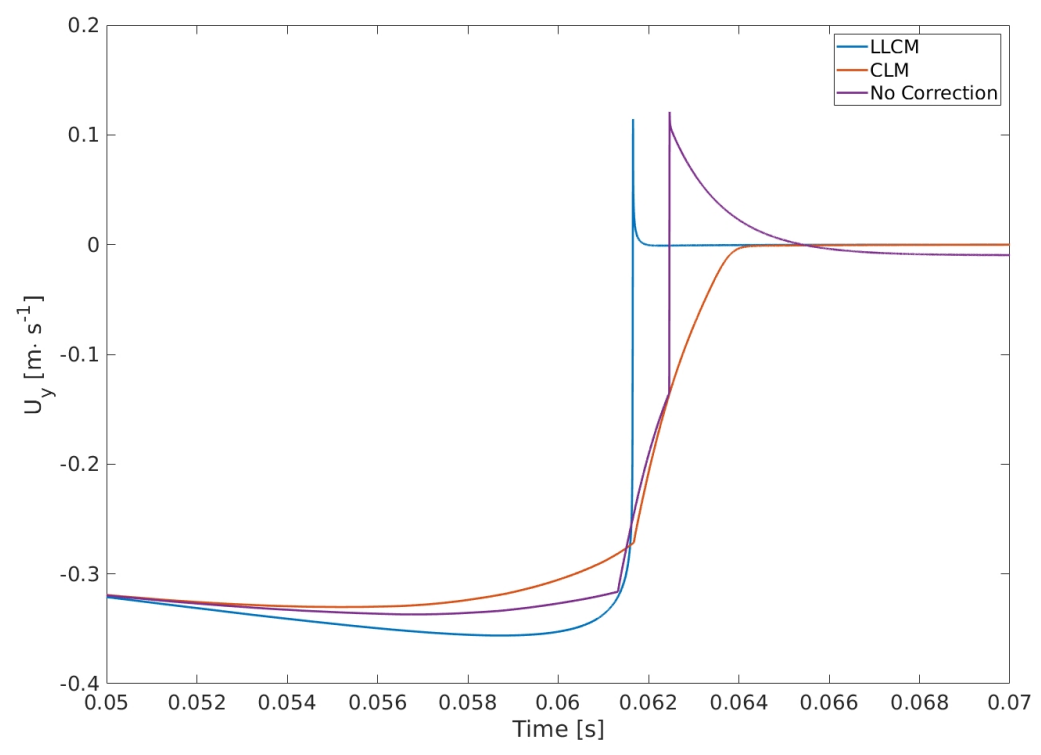

FIGURE 12 Evolution of the particle vertical velocity. Simulations without lubrication correction, with LLCM and CLM are performed using $h=1 / 40$ grid resolution and $\mathcal{A} \mathcal{R}=2$. LLCM and CLM parameters are $\epsilon_{\text {lub }}=2 / h$ and $\epsilon_{\Delta x}=0.06$. 
number $\left(\mathrm{St}_{\mathrm{eq}} \geq 30\right)$ and $\Theta_{\mathrm{z}} \in\left[-5^{\circ}, 5^{\circ}\right]$ have shown that the particle collides with the wall. Therefore, lubrication forces are overestimated with the CLM leading to an unrealistic suspension of the particle above the wall, while LLCM preserves the physics of the system. Due to the lack of analytical or accurate experimental trajectory, a quantitative accuracy of the LLCM cannot be properly assessed.

\section{5 | CONCLUSION AND OUTLOOK}

A numerical method for particle-laden flows of ellipsoidal solid particles using a local lubrication correction model (LLCM) has been presented and validated. Interactions between a particle and an obstacle (another particle or a wall) can be decomposed into three types: long-range hydrodynamics, short-range hydrodynamics also known as lubrication effects, and mechanical solid-solid contacts.

Long range hydrodynamic interactions are fully resolved by the numerical solver based on the Volume Penalization method (VP). The incompressible Navier-Stokes equations have been discretized in time using a scalar projection method and in space with a full second-order penalty method.

Due to unresolved scales associated with the grid, short-range hydrodynamic interactions are only partially captured. Therefore, a local lubrication model is introduced to approximate the unresolved component of the hydrodynamics. This correction is based on asymptotic expansions of analytical solutions of particle-particle or particle-wall interactions, assuming that the flow within the gap between the particle and the obstacle is in the Stokes regime. Lubrication forces and torques are corrected in a neighborhood of the contact point of two interacting particles where lubrication is poorly captured, as long as the normalized gap width $\epsilon$ is smaller than a critical length $\epsilon_{\text {lub }}$ (a model parameter).

Finally, solid contacts are modelled using a linear soft-sphere collision model. This model, widely used in the literature $^{[26,23]}$, represents mechanical contacts as two spring-dashpot systems connected at the contact point. The model allows to stretch the collision time, to avoid computational overhead in the calculation of the collision force, making the method computationally efficient.

Our local lubrication correction model has been validated on several benchmarks for spherical particles in one of our previous works ${ }^{[17]}$. In this article, the method is tested for spheroidal particles with two cases. First, the accuracy of DNS using our numerical framework is evaluated by comparing numerical solutions of isolated spheroids in a sheared flow to analytical solutions. Subsequently, the slow motion of a prolate toward a planar wall is simulated and compared to DNS solution to assess LLCM accuracy. Numerical experiments have shown the LLCM provides more accurate solutions than a similar lubrication model, with a low additional cost ( 10\%). Qualitative comparisons of simulated trajectories with preliminary experimental observations have also shown the LLCM better preserves the physics of the flow compared to existing virtual-sphere-based methods. Due to the lack of analytical or accurate experimental data about the trajectory of the particle, the accuracy of the LLCM could not be quantitatively assessed when the particle collides with a wall. We are looking to address this issue in the future.

\section{ACKNOWLEDGEMENTS}

The simulations presented in this paper were performed on the computing facilities MCIA (Mésocentre de Calcul Intensif Aquitain, on the cluster AVAKAS) of the University of Bordeaux, and on the PLAFRIM experimental parallel test-bed, being developed under the Inria PlaFRIM development action with support from LABRI and IMB and other entities: Conseil Régional d'Aquitaine, FeDER, Université de Bordeaux and CNRS (see https://plafrim.bordeaux.inria.fr/). 


\section{REFERENCES}

[1] Sun R, Xiao H. CFD-DEM Simulations of Current-Induced Dune Formation and Morphological Evolution. Advances in Water Resources 06/2016;92:228-239.

[2] Vowinckel B, Kempe T, Frohlich J, Nikora VI. Numerical simulation of sediment transport in open channel flow. River Flow 01/2012;1:507-514.

[3] Santos A, Bedrikovetsky P. A Stochastic Model for Particulate Suspension Flow in Porous Media. Transport in Porous Media 01/2006;62(1):23-53.

[4] Galaguz YP, Safina GL. Modeling of Particle Filtration in a Porous Medium with Changing Flow Direction. Procedia Engineering 2016;153:157-161.

[5] Kaushal DR, Thinglas T, Tomita Y, Kuchii S, Tsukamoto H. CFD modeling for pipeline flow of fine particles at high concentration. International Journal of Multiphase Flow 2012;43:85-100.

[6] Wan D, Turek S. Direct numerical simulation of particulate flow via multigrid FEM techniques and the fictitious boundary method. International Journal for Numerical Methods in Fluids 06/2006;51(5):531-566.

[7] Usabiaga FB, Kallemov B, Delmotte B, Bhalla APS, Griffith BE, Donev A. Hydrodynamics of Suspensions of Passive and Active Rigid Particles: A Rigid Multiblob Approach. Communications in Applied Mathematics and Computational Science 11/2016;11(2):217-296.

[8] Roussel N, Gram A, editors. Simulation of Fresh Concrete Flow. Springer Netherlands; 2014.

[9] Lefebvre-Lepot A, Merlet B, Nguyen TN. An accurate method to include lubrication forces in numerical simulations of dense Stokesian suspensions. Journal of Fluid Mechanics 2015;769:369-386.

[10] Claeys IL, Brady JF. Suspensions of prolate spheroids in Stokes flow. Part 1. Dynamics of a finite number of particles in an unbounded fluid. Journal of Fluid Mechanics 1993;251:411-442.

[11] Bitsanis I, Somers SA, Davis HT, Tirrell M. Microscopic dynamics of flow in molecularly narrow pores. Journal of Chemical Physics 1990;93(5):3427-3431.

[12] Brady JF, Bossis G. Stokesian dynamics. Annual Review of Fluid Mechanics 01/1988;20:111-157.

[13] Cox RG. The motion of suspended particles almost in contact. International Journal of Multiphase Flow 1974;1:343371.

[14] Staben M, Zinchenko AZ, Davis RH. Dynamic simulation of spheroid motion between two parallel plane walls in lowReynolds-number Poiseuille flow. Journal of Fluid Mechanics 2006;553:187-226.

[15] Ardekani MN, Costa P, Breugem WP, Brandt L. Numerical study of the sedimentation of spheroidal particles. International Journal of Multiphase Flow 12/2016;87:16-34.

[16] Huang H, Yang X, Lu X. Sedimentation of an ellipsoidal particle in narrow tubes. Physics of Fluids 2014;26(5):053302.

[17] Lambert B, Weynans L, Bergmann M. Local lubrication model for spherical particles within incompressible Navier-Stokes flows. Physical Review E 03/2018;97(3).

[18] Angot P, Bruneau CH, Fabrie P. A penalization method to take into account obstacles in incompressible flows. Numerische Mathematik 1999;81(4):497-520.

[19] Mittal R, Dong H, Bozkurttas M, Najjar FM, Vargas A, von Loebbecke A. A versatile sharp interface immersed boundary method for incompressible flows with complex boundaries. Journal of Computational Physics 05/2008;227(10):48254852. 
[20] Chorin AJ. Numerical solution of the Navier-Stokes equations. Mathematics of Computation 1968;22:745-762.

[21] Temam R. Sur l'approximation de la solution des équations de Navier-Stokes par la méthode des pas fractionnaires II. Archive for Rational Mechanics and Analysis 1969;32:377-385.

[22] Lambert B. Modelling and Simulations of Contacts in Particle-Laden Flows. Theses, Université de Bordeaux; 2018.

[23] Izard E, Bonometti T, Lacaze L. Modelling the dynamics of a sphere approaching and bouncing on a wall in a viscous fluid. Journal of Fluid Mechanics 2014;747:422-446.

[24] Jedouaa M, Bruneau CH, Maitre E. An efficient interface capturing method for a large collection of interacting bodies immersed in a fluid. Journal of Computational Physics 2018;378:143-177.

[25] Lin A, Han SP. On the Distance Between Two Ellipsoids. SIAM Journal on Optimization 2002;13(1):298-308.

[26] Costa P, Boersma BJ, Westerweel J, Breugem WP. Collision model for fully-resolved simulations of flows laden with finite-size particles. Physical Review E 10/2015;92(5).

[27] Jeffrey DJ. Low Reynolds Number Flow between Converging Spheres. Mathematika 05/1982;29(1):58-66.

[28] Brenner $\mathrm{H}$. The slow motion of a sphere through a viscous fluid towards a plane surface. Institution of Chemical Engineering Symposium 1961;16:242-251.

[29] Cooley MDA, O'Neill ME. On the Slow Motion Generated in a Viscous Fluid by the Approach of a Sphere to a Plane Wall or Stationary Sphere. Mathematika 1969;16(1):37-49.

[30] Ardekani MN, Costa P, Breugem WP, Picano F, Brandt L. Drag reduction in turbulent channel flow laden with finite-size oblate spheroids. Journal of Fluid Mechanics 01/2017;816:43-70.

[31] de Motta JCB, Breugem WP, Gazanion B, Estivalezes JL, Vincent S, Climent E. Numerical modelling of finite-size particle collisions in a viscous fluid. Physics of Fluids 2013;25:083302.

[32] Kempe T, Fröhlich J. Collision modelling for the interface-resolved simulation of spherical particles in viscous fluids. Journal of Fluid Mechanics 2012;209:445-489.

[33] Zhu C, Yu Z, Shao X. Interface-resolved direct numerical simulations of the interactions between neutrally buoyant spheroidal particles and turbulent channel flows. Physics of Fluids 2018;30(11):115103.

[34] Jeffery GB. The motion of ellipsoidal particles immersed in a viscous fluid. Proceedings of the Royal Society of London A: Mathematical, Physical and Engineering Sciences 01/1922;102(715):161-179.

[35] Lee J, Kim J, Choi H, Yang KS. Sources of spurious force oscillations from an immersed boundary method for moving-body problems. Journal of Computational Physics 2011;230:2677-2695.

\section{GRAPHICAL ABSTRACT}

Lubrication forces are essential in suspensions and are often poorly captured by numerical methods. In this paper, a local lubrication correction model initially developed for spherical particles is extended to ellipsoids. The method has been tested on simple numerical experiments and solutions have been compared to preliminary in-lab measurements. 